\title{
Predictive factors for delayed hyponatremia after transsphenoidal surgery in patients with pituitary adenomas
}

\author{
Kunzhe Lin ${ }^{1,2,{ }^{*}}$, Lingling Lu ${ }^{2, *}$, Zhijie Pei ${ }^{2}$, Shuwen $\mathrm{Mu}^{2}$, Shaokuan Huang ${ }^{3}$ and Shousen Wang ${ }^{2,4}$ \\ ${ }^{1}$ Department of Neurosurgery, Affiliated Fuzhou First Hospital of Fujian Medical University, Fuzhou, China \\ 2Fuzong Clinical Medical College of Fujian Medical University, Fuzhou, China \\ ${ }^{3}$ Department of Neurosurgery, Guiqian International General Hospital, Guiyang, China \\ ${ }^{4}$ Department of Neurosurgery, 900th Hospital, Fuzhou, China \\ Correspondence should be addressed to S Huang or S Wang: 1123228680@qq.com or wshsen1965@126.com \\ *(K Lin and L Lu contributed equally to this work)
}

\begin{abstract}
Objective: The aim of this study was to evaluate the incidence and duration of delayed hyponatremia and to assess the factors influencing the development of delayed hyponatremia after transsphenoidal surgery (TSS) in pituitary adenomas.

Methods: We retrospectively analyzed the clinical data of patients with pituitary adenoma who underwent TSS. Univariable and multivariable statistics were carried out to identify factors independently associated with the occurrence of delayed hyponatremia.

Results: Of the 285 patients with pituitary adenoma who underwent microscopic TSS, 44 (15.4\%) developed postoperative-delayed hyponatremia and 241 (84.6\%) did not. The onset of delayed hyponatremia occurred an average of 5.84 days post-surgery and persisted for an average of 5.36 days. Logistic regression analysis showed the highest risk of delayed hyponatremia in patients with significant change in tumor cavity height (odds ratio (OR), 1.158; $95 \% \mathrm{Cl}, 1.062,1.262 ; P=0.001)$, preoperative hypothalamuspituitary-thyroid axis hypofunction (OR, 3.112; $95 \% \mathrm{Cl}, 1.481,6.539 ; P=0.003)$, and significant difference in blood sodium levels before and 2 days after TSS (OR, 1.101; 95\% Cl, 1.005, 1.206; $P=0.039$ ).

Conclusions: Preoperative hypothyroidism, difference in blood sodium levels before and 2 days after TSS, and the change in tumor cavity height after TSS played important roles in predicting postoperative-delayed hyponatremia onset in patients with pituitary adenomas.
\end{abstract}

\author{
Key Words \\ - hyponatremia \\ - hypothyroidism \\ - pituitary adenoma \\ - transsphenoidal surgery
}

\section{Introduction}

Delayed hyponatremia refers to hyponatremia that occurs $\geq 3$ days after transsphenoidal surgery (TSS) (1). Patients with hyponatremia may have various clinical manifestations, ranging from asymptomatic to mild (headache, nausea, and vomiting) or severe symptoms. Severe hyponatremia can cause altered levels of consciousness, cerebral edema, seizures, and even death ( 2 , $3)$. Delayed hyponatremia is often ignored by clinicians, but it is the main cause of readmission $(4,5)$. The most common cause of delayed hyponatremia is the syndrome of inappropriate antidiuretic hormone secretion (SIADH), while cerebral salt wasting syndrome is a rare cause (6).

Following TSS for pituitary tumors, patients are at high risk of developing water balance disorders, including diabetes insipidus and delayed hyponatremia. The management of delayed hyponatremia is challenging, as there is limited understanding of the predictive factors of its occurrence. Moreover, its clinical treatment is https://ec.bioscientifica.com

https://doi.org/10.1530/EC-21-0497 (c) 2022 The authors Published by Bioscientifica Ltd

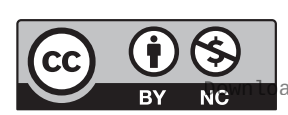

This work is licensed under a Creative Commons Attribution-NonCommercial 4.0 International License. ded from Bioscientifica.com at 04/26/2023 09:55:35AM 
complex, and most treatments require close observation and frequent laboratory testing in the intensive care unit, resulting in high hospital costs (7). Therefore, screening and identifying patients at high risk for delayed hyponatremia after surgery, in addition to close monitoring and timely treatment, are crucial. Previous studies reported old age, low sodium concentration on postoperative days 1-2, long operation duration, low BMI, younger age, and lower preoperative serum sodium as predictors of delayed hyponatremia $(3,6,8)$. However, delayed hyponatremia is generally believed to be caused by mechanical damage to the hypothalamusneurohypophyseal system caused by TSS. Unfortunately, clinicians cannot modify the above factors to reduce the degree of injury and the incidence of postoperativedelayed hyponatremia.

The present study evaluated delayed hyponatremia incidence and duration after pituitary adenoma resection via microscopic TSS and identified the predictive factors affecting its occurrence.

\section{Methods}

\section{Patient cohort}

This study retrospectively analyzed clinical data of patients with pituitary adenoma who underwent TSS in our Neurosurgery Department between January 2017 and December 2020. This study was approved by the 900th hospital Institutional Review Board (2019-017). The patients gave consent to use their clinical data for research purposes, and all data were anonymized.

We included (i) patients who underwent microscopic TSS for pituitary adenomas; (ii) patients who were operated on by the same surgeon; and (iii) cases in which the tumor grew upward and through the tuberculum sellae-dorsum sellae plane. We excluded (i) patients with pathologically confirmed non-pituitary adenoma or pituitary adenoma with other concomitant sellar lesions and (ii) cases in which the tumor growth did not reach the tuberculum sellae-dorsum sellae plane.

\section{MRI evaluation}

The pituitary gland was scanned using a 3.0-T MRI scanner (Tim Trio; Siemens Medical Solutions). Images were evaluated to identify potential factors for postoperativedelayed hyponatremia, including tumor cavity height (preoperative and postoperative), tumor volume, tumor volume of the superior part, invasiveness, intratumoral cysts or hematoma, location of the posterior pituitary bright spot (PPBS), and extent of tumor resection. The tumor was divided into superior and inferior parts using the tuberculum sellae-dorsum sellae plane as the boundary (9). The tumor cavity height was measured pre- and postoperatively at the same coronal position on contrast-enhanced images, and the difference between the two was taken as the change in tumor cavity height after TSS (Fig. 1). Patients with Knosp grades 3 and 4 were identified as having cavernous sinus invasion (10). All patients underwent routine postoperative MRI at 1-3 days and 4-6 months to evaluate the extent of tumor resection. The extent of tumor resection was classified as: gross total resection, without any evidence of residual adenoma; subtotal resection, residual adenoma $<20 \%$; and partial resection, residual adenoma $<50 \%$ (11). Tumor volume was calculated using the platform-like volume calculation formula (12). The magnetic resonance images were independently evaluated and measured by
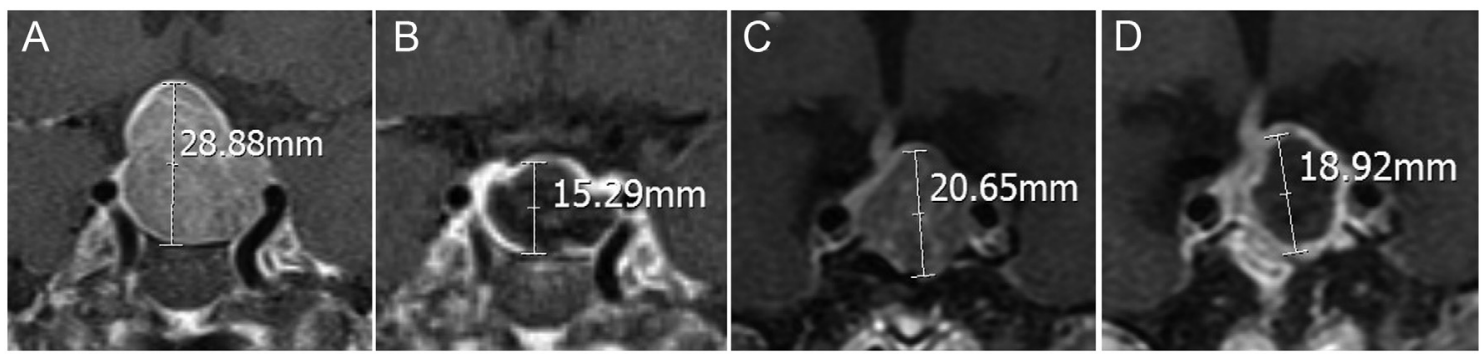

\section{Figure 1}

Coronal contrast-enhanced images of the change in tumor cavity height after transsphenoidal surgery in two cases. Panels A and B and panels C and D are from the same patient, respectively. (A) Before surgery, the tumor cavity height is $28.88 \mathrm{~mm}$. (B) After surgery, the tumor cavity height is $15.29 \mathrm{~mm}$, and the change in tumor cavity height after transsphenoidal surgery of this patient is $28.88-15.29 \mathrm{~mm}$. (C) Before surgery, the tumor cavity height is $20.65 \mathrm{~mm}$. (D) After surgery, the tumor cavity height is $18.92 \mathrm{~mm}$, and the change in tumor cavity height after transsphenoidal surgery of this patient is $20.65-18.92 \mathrm{~mm}$ 
two neurosurgeons. In cases in which the measurement differed by $>20 \%$ among the reviewers, a consensus was reached through a discussion and the mean of the measurements was used for the statistical analysis.

\section{Perioperative evaluations}

All patients have serum sodium levels checked every day and received sodium chloride solution $(0.9 \%)$ as maintenance fluid postoperatively. Preoperative routine examination of the hypothalamus-pituitary-adrenal (HPA) axis and hypothalamus-pituitary-thyroid (HPT) axis functions was performed to determine the low function and the need for oral glucocorticoid and thyroxine replacement therapy. Intraoperative stress doses of glucocorticoids were administered intravenously to all patients (except to those with Cushing's disease) to prevent the rapid surgery-related decline in HPA axis functions. Patients with HPA axis and HPT axis dysfunction were given glucocorticoid and thyroxine replacement therapy. On the first postoperative day, patients fasted and were given i.v. fluids of 2000-2500 $\mathrm{mL}$. The patients were allowed to start on a liquid diet the day after surgery, with a gradual transition to a semiliquid diet depending on the patient's condition. Patients with comorbidities (e.g. cardiac insufficiency, diabetes) should be treated with individualized fluid rehydration to maintain energy needs. Patients with diabetes insipidus should drink water to relieve thirst, and oral desmopressin was given if necessary. For patients with delayed hyponatremia, the cause needs to be determined carefully. If delayed hyponatremia is caused by SIADH, fluid intake should be restricted. If the cause is cerebral salt wasting syndrome, treatment is administered to correct the low blood volume and to provide sodium supplementation.

The patient's age, sex, MRI features, intraoperative cerebrospinal fluid leakage, pathological tumor type, HPT axis function, HPA axis function, and electrolyte levels were recorded. The criteria for HPT and HPA hypofunction were based on Bordo et al. (2).

\section{Statistical analysis}

All statistical analyses were performed using IBM SPSS Statistics for Windows, version 20.0 (IBM Corp.). The clinical data were expressed as mean and S.D.; median, interquartile range; and frequency and percentage. Independent samples $t$-test, Wilcoxon rank-sum test, Fisher's exact test, chi-squared test, and logistic regression analysis were applied as appropriate.
Table 1 Demographic characteristics of 285 patients who underwent transsphenoidal surgery for pituitary adenomas.

\begin{tabular}{lc}
\hline Variable & No. \\
\cline { 1 - 2 } Age, mean \pm S.D., years & $49.2 \pm 12.9$ \\
Sex & 146 \\
Male & 139 \\
Female & $6.6 \pm 6.4$ \\
Tumor size, cm ${ }^{3}$ & $25.7 \pm 8.5$ \\
Tumor height, mm & \\
Pathological tumor type & 93 \\
Gonadotropinoma & 78 \\
Null cell adenoma & 41 \\
PRL cell adenoma & 19 \\
GH cell adenoma & 24 \\
ACTH cell adenoma & 28 \\
Plurihormonal & 2 \\
TSH cell adenoma & \\
Intratumoral cysts or hematoma & 115 \\
Yes & 170 \\
No & \\
Location of the PPBS & 146 \\
Superior parts & 97 \\
Inferior parts & 13 \\
Superior and inferior parts & 29 \\
None & \\
Invasiveness & 44 \\
Yes & 241 \\
No & \\
\hline
\end{tabular}

ACTH, adrenocorticotropic hormone; GH, growth hormone; $\mathrm{TSH}$, thyroid-stimulating hormone; PPBS, posterior pituitary bright spot; PRL, prolactin.

\section{Results}

\section{Participant characteristics}

This study included a total of 285 eligible patients with pituitary adenomas (Table 1). Our cohort included 146 men and 139 women, with a mean age of $49.2 \pm 12.9$ years. Forty-four cases (15.4\%) developed postoperative-delayed hyponatremia, while 241 cases $(84.6 \%)$ did not. The onset of hyponatremia occurred at an average of 5.84 days postsurgery and persisted for an average of 5.36 days. The lowest average blood sodium level was $123.6 \mathrm{mEq} / \mathrm{L}$, which occurred 7.88 days after surgery.

\section{Factors influencing postoperative-delayed hyponatremia}

Table 2 summarizes the results of comparisons between the delayed hyponatremia and normonatremia groups. The change in tumor cavity height was significantly larger in patients with delayed hyponatremia compared to those without delayed hyponatremia $(P<0.001)$. The postoperative tumor cavity height of the delayed

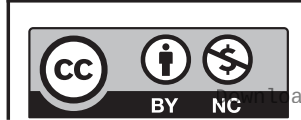

This work is licensed under a Creative Commons Attribution-NonCommercial 4.0 International License. ded from Bioscientifica.com at 04/26/2023 09:55:35AM 
Table 2 Univariate analysis of postoperative-delayed hyponatremia.

\begin{tabular}{|c|}
\hline Factors \\
\hline Age \\
\hline Sex \\
\hline Male \\
\hline Female \\
\hline Tumor volume, $\mathrm{cm}^{3}$ \\
\hline Tumor volume of superior part, $\mathrm{cm}^{3}$ \\
\hline Tumor cavity height (preoperative), mm \\
\hline Tumor cavity height (postoperative), mm \\
\hline Pathological tumor type \\
\hline Gonadotropinoma \\
\hline Null cell adenoma \\
\hline PRL cell adenoma \\
\hline GH cell adenoma \\
\hline ACTH cell adenoma \\
\hline Plurihormona \\
\hline TSH cell adenoma \\
\hline Intratumoral cysts or hematoma \\
\hline Yes \\
\hline No \\
\hline Location of the PPBS \\
\hline Superior parts \\
\hline Inferior parts \\
\hline Superior and inferior parts \\
\hline None \\
\hline Invasiveness \\
\hline Yes \\
\hline No \\
\hline Preoperative HPT axis function \\
\hline Hypofunction \\
\hline Normofunction \\
\hline Preoperative HPA axis function \\
\hline Hypofunction \\
\hline Normofunction \\
\hline Extent of tumor resection \\
\hline Total resection \\
\hline Subtotal resection \\
\hline Partial resection \\
\hline Intraoperative cerebrospinal fluid leaks \\
\hline Yes \\
\hline No \\
\hline Change in tumor cavity height, $\mathrm{mm}$ \\
\hline Postoperative HPT axis function \\
\hline Hypofunction \\
\hline Normofunction \\
\hline Postoperative HPA axis function \\
\hline Hypofunction \\
\hline Normofunction \\
\hline
\end{tabular}

\begin{tabular}{c}
\hline Delayed hyponatremia \\
\hline $41(49,58.7)$ \\
23 \\
21 \\
$5.4(3.5,11.1)$ \\
$1.5(0.5,2.7)$ \\
$22.8(18.9,26.5)$ \\
$15.3(12,18.4)$
\end{tabular}

$15.3(12,18.4)$

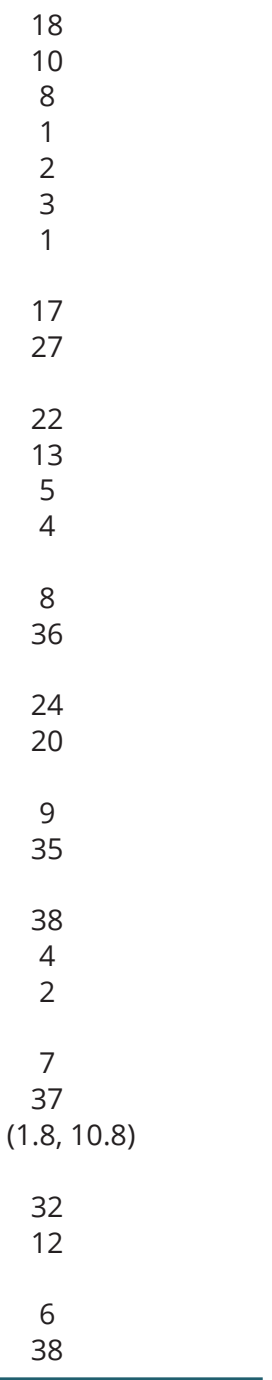

\begin{tabular}{ccc}
\hline Normonatremia & & \multicolumn{1}{c}{$\boldsymbol{P}$ value } \\
$(40.5,58.5)$ & & 0.901 \\
& & 0.92 \\
124 & & \\
117 & & 0.126 \\
$4.3(2.5,7.6)$ & & 0.155 \\
$1.1(0.4,2.1)$ & & 0.195 \\
$21.2(18.6,25.5)$ & & 0.01 \\
$17.9(13.8,21.3)$ & & 0.333
\end{tabular}

0.801

0.964

$<0.001$

$<0.001$

0.523

ACTH, adrenocorticotropic hormone; GH, growth hormone; HPA, hypothalamus-pituitary-adrenal; HPT, hypothalamus-pituitary-thyroid; PPBS, posterior pituitary bright spot; PRL, prolactin; TSH, thyroid-stimulating hormone.

hyponatremia group was significantly lower than that of the normonatremia group $(P=0.01)$. We also observed a significant difference in the number of patients with preoperative and postoperative HPT axis hypofunction between the two groups $(P<0.001$ for both). We observed no significant differences between the delayed hyponatremia and normonatremia groups in terms of age, sex, immunohistochemical types, PPBS location, tumor cystic degeneration or hemorrhage, and pre- and postoperative HPA function.

Patients with and without postoperative-delayed hyponatremia showed no significant differences in blood sodium levels preoperatively and at 1 and 2 days postTSS. However, the delayed hyponatremia group showed

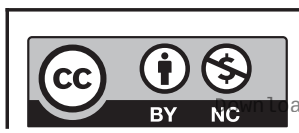

This work is licensed under a Creative Commons Attribution-NonCommercial 4.0 International License. ded from Bioscientifica.com at 04/26/2023 09:55:35AM via free access 
Table 3 Analysis of the difference of blood sodium before and after TSS.

\begin{tabular}{l} 
Factors \\
\hline Preoperative serum sodium, $\mathrm{mEq} / \mathrm{L}$ \\
Blood sodium on the first day after surgery, $\mathrm{mEq} / \mathrm{L}$ \\
Serum sodium on the second day after surgery, $\mathrm{mEq} / \mathrm{L}$ \\
The difference in blood sodium levels before and 1 days \\
after TSS, $\mathrm{mEq} / \mathrm{L}$ \\
The difference in blood sodium levels before and 2-day \\
after TSS, $\mathrm{mEq} / \mathrm{L}$
\end{tabular}

\begin{tabular}{c}
\hline Delayed hyponatremia \\
\hline $140.9(139.2,143)$ \\
$140.7(137.5,142.3)$ \\
$139.7(137.4,142.7)$ \\
$0.85(-1.17,3.0)$ \\
$1.4(-2.4,3.35)$
\end{tabular}

TSS, transsphenoidal Surgery.

greater differences from the preoperative levels than the normonatremia group $(P=0.026)$, in which patients with postoperative-delayed hyponatremia group experienced substantial declines in blood sodium levels at 2 days postTSS (Table 3).

\section{Risk of postoperative hyponatremia onset}

Based on the above results of univariate analyses, we selected the following variables with $P<0.05$ for logistic regression analysis: the difference in blood sodium levels before and 2 days after TSS, change in tumor cavity height, postoperative tumor cavity height, and preoperative and postoperative HPT axis function. This analysis showed a higher risk of delayed hyponatremia in patients with a significant change in tumor cavity height (odds ratio (OR)], 1.158; 95\% CI, 1.062, 1.262; $P=0.001$ ), preoperative HPT axis hypofunction (OR, 3.112; 95\% CI, 1.481, 6.539; $P=0.003$ ), and a difference in blood sodium levels before and 2 days after TSS (OR, 1.101; 95\% CI, 1.005, 1.206; $P=0.039$ ) (Table 4).

\section{Discussion}

This clinical study investigated the incidence and predictors of delayed hyponatremia after TSS. Our results demonstrated that the incidence of delayed hyponatremia was $15.4 \%$. Furthermore, the independent predictors of postoperative-delayed hyponatremia onset were changes in tumor cavity height after TSS, preoperative HPT axis function, and the difference in blood sodium levels before and 2 days after TSS.

Previous studies reported that the incidence rate of delayed hyponatremia after TSS for pituitary adenomas is between 4 and $23 \%$ (6). The main clinical manifestation of SIADH is delayed hyponatremia due to the uncontrolled release of antidiuretic hormone secretion (ADH) following damage to the hypothalamus-neurohypophyseal system, leading to water retention. We identified predictors of delayed hyponatremia that are different from those reported previously. Age (young and old) has been identified as a predictor of postoperative-delayed hyponatremia $(3,6$, 13). Moreover, owing to the sex-specific difference in the response to antidiuretic hormone, women are more likely than men to develop postoperative-delayed hyponatremia (14). Another study reported that the larger the tumor, the more the disruption of the pituitary stalk during surgery and the more likely the development of delayed hyponatremia after surgery (13). Our study found that age, sex, and tumor size did not predict the occurrence of delayed hyponatremia after surgery.

Pituitary adenomas can extend into the cavernous sinus, suprasellar space, sphenoid sinus, or clivus (15). When a pituitary adenoma grows, it may compress the pituitary stalk, resulting in its chronic distortion and may lead to changes in the position of the posterior pituitary gland (i.e. an ectopic posterior pituitary). The display rate and location of the PPBS of patients with pituitary adenoma can be clearly visualized via MRI $(16,17)$. The presence of

Table 4 Logistic regression analysis of the risk of postoperative-delayed hyponatremia onset.

\begin{tabular}{|c|c|}
\hline \multicolumn{2}{|l|}{ Factors } \\
\hline \multirow{5}{*}{\multicolumn{2}{|c|}{$\begin{array}{l}\text { Change in tumor cavity height after TSS } \\
\text { Postoperative tumor cavity height } \\
\text { Preoperative HPT axis function } \\
\text { Postoperative HPT axis function } \\
\text { The difference in blood sodium levels before and 2-day after TSS }\end{array}$}} \\
\hline & \\
\hline & \\
\hline & \\
\hline & \\
\hline \multicolumn{2}{|c|}{ OR, odds ratio; HPT, hypothalamus-pituitary-thyroid; TSS, transsphenoidal s } \\
\hline $\begin{array}{l}\text { https://ec.bioscientifica.com } \\
\text { https://doi.org/10.1530/EC-21-0497 }\end{array}$ & $\begin{array}{r}\text { (c) } 2022 \text { The authors } \\
\text { Published by Bioscientifica Ltd }\end{array}$ \\
\hline
\end{tabular}

\begin{tabular}{c}
\hline Odds ratio \\
\hline 1.158 \\
0.984 \\
3.112 \\
0.883 \\
1.101 \\
\hline
\end{tabular}

\begin{tabular}{c}
\hline $\mathbf{9 5 \%} \mathbf{C l}$ \\
\hline $1.062,1.262$ \\
$0.908,1.066$ \\
$1.481,6.539$ \\
$0.392,1.989$ \\
$1.005,1.206$ \\
\hline
\end{tabular}

\begin{tabular}{c}
\hline $\boldsymbol{P}$ value \\
\hline 0.001 \\
0.688 \\
0.003 \\
0.764 \\
0.039 \\
\hline
\end{tabular}

al surgery. 
PPBS reflects the accumulation of $\mathrm{ADH}$, which is a sign of the functional integrity of the neurohypophysis; however, its absence does not necessarily imply impaired function of the neurohypophysis (18). Therefore, in patients with pituitary adenomas, diabetes insipidus or SIADH is rarely seen before surgery. In other words, for pituitary adenomas with different growth directions, there is no abnormal $\mathrm{ADH}$ secretion before surgery. However, after the tumor resection, observing the changes in the PPBS and pituitary stalk may be helpful to determine whether there is a change in $\mathrm{ADH}$ secretion; this requires further research.

Jahangiri reported that preoperative hypopituitarism was a sign of the mechanical manipulation of the pituitary stalk and posterior lobe of the pituitary gland during surgery and was, therefore, a potential risk factor for the occurrence of postoperative hyponatremia (19). The results of the multivariate analysis in the present study suggested that preoperative hypothyroidism was an independent predictor of postoperative-delayed hyponatremia. Hypothyroidism can cause hyponatremia by increasing $\mathrm{ADH}$ levels and intrarenal mechanisms (2). Low thyroxine levels cause decreased cardiac output, stimulate baroreceptors in the carotid sinus, and release $\mathrm{ADH}$. Moreover, low thyroxine levels can also reduce the glomerular filtration rate and the excretion of free water by reducing the original urine volume delivered to the distal tubules and collecting ducts (20). Therefore, for patients with hypothyroidism before surgery, appropriate thyroxine supplementation may be used to reduce the occurrence of postoperative hyponatremia.

SIADH-related hyponatremia after TSS usually occurs approximately 5-7 days after surgery, appearing on average on the sixth day $(1,8,21)$. Patients with serum sodium concentrations $<138 \mathrm{mEq} / \mathrm{L}$ after $1-2$ days are more likely to develop delayed hyponatremia (3). Krogh et al. (5) also reported an increased risk of hyponatremia in patients with decreased blood sodium concentrations on the first day after surgery. Our results suggest that compared to preoperative serum sodium, a large decrease in serum sodium concentration 2 days post-TSS is an independent predictor of delayed hyponatremia. We speculate that the abnormal secretion of ADH may occur from the second day after TSS, despite blood sodium concentrations being within the normal range.

Owing to the limited field of vision during the operation, none of the previous predictors of delayed hyponatremia involved damage assessment. Recently, some researchers have proposed that diaphragma sellae descent could be a cause of hypothalamus-neurohypophysis tract injury, which further leads to SIADH after pituitary surgery (22). However, patients with thin or no diaphragma sellae showed no descent of the diaphragma sellae. We measured the height of the tumor cavity before and after TSS and found that the difference in tumor cavity height predicted the occurrence of delayed hyponatremia after surgery. One advantage of this study was that it was easier to observe and measure the height of the tumor cavity than the height of the diaphragma sellae. The difference in tumor cavity height before and after TSS reflected the sinking depth of the saddle diaphragm, tumor capsule, arachnoid, or flat pituitary tissue above the pituitary tumor owing to tumor removal. This sinking pulls, changes the position and shape, and damages the pituitary stalk, further leading to SIADH. Reducing the tumor cavity's height change may help lessen damage to the pituitary stalk. Therefore, with the gradual removal of the tumor during surgery, the tumor cavity should be filled with a certain volume of gelatin sponge to prevent lowering of the saddle diaphragm to reduce the change in height of the tumor cavity.

The diaphragma sellae can be elevated up to or beyond the level of the corpus callosum by the growing tumor $(23,24)$. The surgical removal of the tumor makes the diaphragma sellae sink, indicating that the operation can be terminated. For tumors with cystic changes, the outflow of cystic fluid causes the intrasellar pressure to decrease sharply, the sellar diaphragm descends, and the height of the tumor cavity decreases rapidly. However, after we pack the gelatin sponge into the tumor cavity, the sellar diaphragm can be elevated to reduce the change in the tumor cavity height after TSS, thereby reducing the degree of traction of the pituitary stalk. This study found no difference in the incidence of postoperatively delayed hyponatremia between cystic and non-cystic patients. If there is little or no intrasellar packing, the height of the tumor cavity changes greatly after the operation, and the pituitary stalk exhibits a process of continuous pulling that leads to abnormal $\mathrm{ADH}$ secretion and delayed hyponatremia.

\section{Conclusion}

The results of this study provide evidence of the important role of changes in tumor cavity height before and after TSS in predicting the onset of postoperative-delayed hyponatremia in patients with pituitary adenomas. We also showed that patients with preoperative hypothyroidism had a risk of delayed hyponatremia. The difference in blood sodium levels before and 2 days after

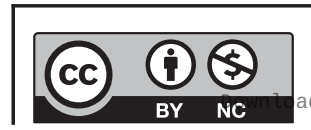

This work is licensed under a Creative Commons Attribution-NonCommercial 4.0 International License. ded from Bioscientifica.com at 04/26/2023 09:55:35AM 
TSS was an independent predictor of postoperative-delayed hyponatremia onset.

\section{Declaration of interest}

The authors declare that there is no conflict of interest that could be perceived as prejudicing the impartiality of the research reported.

\section{Funding}

This work was supported by the Natural Science Foundation of Fujian Province (2021J011306), the Department of Science and Technology of Fuzhou City (2021-S-180), and Startup Fund for scientific research of Fujian Medical University (2018QH1244).

\section{References}

1 Patel KS, Shu Chen J, Yuan F, Attiah M, Wilson B, Wang MB, Bergsneider M \& Kim W. Prediction of post-operative delayed hyponatremia after endoscopic transsphenoidal surgery. Clinical Neurology and Neurosurgery 2019182 87-91. (https://doi.org/10.1016/j.

2 Bordo G, Kelly K, McLaughlin N, Miyamoto S, Duong HT, Eisenberg A, Chaloner C, Cohan P, Barkhoudarian G \& Kelly DF. Sellar masses that present with severe hyponatremia. Endocrine Practice 201420 1178-1186. (https://doi.org/10.4158/EP13370.OR)

3 Yoon HK, Lee HC, Kim YH, Lim YJ \& Park HP. Predictive factors for delayed hyponatremia after endoscopic transsphenoidal surgery in patients with nonfunctioning pituitary tumors: a retrospective observational study. World Neurosurgery 2019122 e1457-e1464. (https://doi.org/10.1016/j.wneu.2018.11.085)

4 Bohl MA, Ahmad S, Jahnke H, Shepherd D, Knecht L, White WL \& Little AS. Delayed hyponatremia is the most common cause of 30-day unplanned readmission after transsphenoidal surgery for pituitary tumors. Neurosurgery $2016 \mathbf{7 8}$ 84-90. (https://doi.org/10.1227/ NEU.0000000000001003)

5 Krogh J, Kistorp CN, Jafar-Mohammadi B, Pal A, Cudlip S \& Grossman A. Transsphenoidal surgery for pituitary tumours: frequency and predictors of delayed hyponatraemia and their relationship to early readmission. European Journal of Endocrinology 2018178 247-253. (https://doi.org/10.1530/EJE-17-0879)

6 Sorba EL, Staartjes VE, Voglis S, Tosic L, Brandi G, Tschopp O, Serra C \& Regli L. Diabetes insipidus and syndrome of inappropriate antidiuresis (SIADH) after pituitary surgery: incidence and risk factors. Neurosurgical Review 202144 1503-1511. (https://doi.org/10.1007/s10143-020-01340-0)

7 Winograd D, Staggers KA, Sebastian S, Takashima M, Yoshor D \& Samson SL. An effective and practical fluid restriction protocol to decrease the risk of hyponatremia and readmissions after transsphenoidal surgery. Neurosurgery 202087 761-769. (https://doi.org/10.1093/neuros/nyz555)

8 Matsuyama J, Ikeda H, Sato S, Yamamoto K, Ohashi G \& Watanabe K. Early water intake restriction to prevent inappropriate antidiuretic hormone secretion following transsphenoidal surgery: low BMI predicts postoperative SIADH. European Journal of Endocrinology 2014 171 711-716. (https://doi.org/10.1530/EJE-14-0530)

9 Lin K, Li J, Lu L, Zhang S, Mu S, Pei Z, Wang C, Lin J, Xue L, Wei L, et al. Diaphragma sellae sinking can predict the onset of hyponatremia after transsphenoidal surgery for pituitary adenomas. Journal of Endocrinological Investigation 202144 2511-2520. (https://doi. org/10.1007/s40618-021-01611-7)

10 Knosp E, Steiner E, Kitz K \& Matula C. Pituitary adenomas with invasion of the cavernous sinus space: a magnetic resonance imaging clineuro.2019.05.007)

classification compared with surgical findings. Neurosurgery 1993 33 610-617; discussion 617-618. (https://doi.org/10.1227/00006123199310000-00008)

11 Karki M, Sun J, Yadav CP \& Zhao B. Large and giant pituitary adenoma resection by microscopic trans-sphenoidal surgery: surgical outcomes and complications in 123 consecutive patients. Journal of Clinical Neuroscience 201744 310-314. (https://doi.org/10.1016/j. jocn.2017.07.015)

12 Wang S, Lin S, Wei L, Zhao L \& Huang Y. Analysis of operative efficacy for giant pituitary adenoma. BMC Surgery 201414 59. (https://doi. org/10.1186/1471-2482-14-59)

13 Lee JI, Cho WH, Choi BK, Cha SH, Song GS \& Choi CH. Delayed hyponatremia following transsphenoidal surgery for pituitary adenoma. Neurologia Medico-Chirurgica 200848 489-492; discussion 492-494. (https://doi.org/10.2176/nmc.48.489)

14 Barber SM, Liebelt BD \& Baskin DS. Incidence, etiology and outcomes of hyponatremia after transsphenoidal surgery: experience with 344 consecutive patients at a single tertiary center. Journal of Clinical Medicine 20143 1199-1219. (https://doi.org/10.3390/jcm3041199)

15 Zada G, Lin N \& Laws Jr ER. Patterns of extrasellar extension in growth hormone-secreting and nonfunctional pituitary macroadenomas. Neurosurgical Focus 201029 E4. (https://doi.org/10.3171/2010.7.FO CUS10155)

16 Saeki N, Tokunaga H, Wagai N, Sunami K, Murai H, Kubota M, Tatsuno I, Saito Y \& Yamaura A. MRI of ectopic posterior pituitary bright spot with large adenomas: appearances and relationship to transient postoperative diabetes insipidus. Neuroradiology $2003 \mathbf{4 5}$ 713-716. (https://doi.org/10.1007/s00234-003-1018-9)

17 Saeki N, Hayasaka M, Murai H, Kubota M, Tatsuno I, Takanashi J, Uno T, Iuchi T \& Yamaura A. Posterior pituitary bright spot in large adenomas: MR assessment of its disappearance or relocation along the stalk. Radiology 2003226 359-365. (https://doi.org/10.1148/ radiol.2262011616)

18 Bonneville F, Narboux Y, Cattin F, Rodière E, Jacquet G \& Bonneville JF. Preoperative location of the pituitary bright spot in patients with pituitary macroadenomas. American Journal of Neuroradiology 200223 528-532.

19 Jahangiri A, Wagner J, Tran MT, Miller LM, Tom MW, Kunwar S, Blevins Jr L \& Aghi MK. Factors predicting postoperative hyponatremia and efficacy of hyponatremia management strategies after more than 1000 pituitary operations. Journal of Neurosurgery 2013119 1478-1483. (https://doi.org/10.3171/2013.7.JNS13273)

20 Katoch CD, Brar KS \& Singh B. Evaluation of thyroid and adrenal functions in patients with hyponatremia. Medical Journal, Armed Forces India 201369 237-240. (https://doi.org/10.1016/j.mjafi.2013.01.003)

21 Kristof RA, Rother M, Neuloh G \& Klingmüller D. Incidence, clinical manifestations, and course of water and electrolyte metabolism disturbances following transsphenoidal pituitary adenoma surgery: a prospective observational study. Journal of Neurosurgery 2009111 555-562. (https://doi.org/10.3171/2008.9.JNS08191)

22 Lin K \& Wang S. Letter: Focus on syndrome of inappropriate antidiuretic hormone after resection of pituitary lesions via transsphenoidal approach. Neurosurgery 202189 E80-E81. (https://doi. org/10.1093/neuros/nyab110)

23 Goel A, Nadkarni T, Muzumdar D, Desai K, Phalke U \& Sharma P. Giant pituitary tumors: a study based on surgical treatment of 118 cases. Surgical Neurology 200461 436-445; discussion 445-446. (https://doi. org/10.1016/j.surneu.2003.08.036)

24 Goel A. Correspondence: Pituitary tumors and diaphragma sellae. Re: Cabuk B, Anik I, Kokturk S, Ceylan S, Ceylan S: Anatomic and histologic features of diaphragma sellae that effects the suprasellar extension. Journal of Clinical Neuroscience 71(2020) 234-244. Journal of Clinical Neuroscience 202077 253. (https://doi.org/10.1016/j.jocn.2020.04.093)

Received in final form 12 November 2021

Accepted 3 December 2021

Accepted Manuscript published online 3 December 2021
This work is licensed under a Creative Commons Attribution-NonCommercial 4.0 International License. ded from Bioscientifica.com at 04/26/2023 09:55:35AM 